\title{
Claves para dinamizar una asignatura básica de matemáticas, utilizando materiales disponibles en la web
}

\author{
Amanda Carreño $^{a}$, Mario Gimeno Soriano ${ }^{b}$, Esther Sanabria Codesal ${ }^{c}$ y David Sixto $^{b}$ \\ anstituto Universitario de Seguridad Industrial, Radiofísica y Medioambiental, Universitat \\ Politècnica de València (amcarsan@iqn.upv.es), b Escuela Técnica Superior de Ingeniería del Diseño, \\ Universitat Politècnica de València (․magiso5@etsid.upv.es, dasixna@etsid.upv.es), ${ }^{c}$ Departamento de \\ Matemática Aplicada, Universitat Politècnica de València (esanabri@mat.upv.es)
}

\begin{abstract}
One of the ways to increase students' motivation and participation in the classroom is to propose a wide variety of resources and activities so that the teaching materials to be used are suitable for the largest possible number of students. Today's students have grown up in an environment where technological supports are part of their daily lives. For this reason, this work proposes the incorporation of Information and Communication Technologies (TIC) together with other quality materials as a medium for information on teaching content. The main objective of this methodology is that the proposed tools allow learning the subject in a more attractive and enjoyable way, as well as promoting communication between students and teachers. This model has been implemented within the Mathematics I classes of the Degree in Industrial Electronic and Automatic Engineering, both in theory and in classroom and computer practices.
\end{abstract}

Keywords: TIC, Technology Resources, Math Learning, Dynamic Materials

\footnotetext{
Resumen

Una de las maneras de aumentar la motivación y la participación en las aulas por parte de los alumnos es el planteamiento de una gran variedad de recursos y actividades de forma que los materiales docentes a trabajar se adecuen al mayor número de alumnos posible. Los alumnos de hoy en día han crecido en un ámbito donde los soportes tecnológicos forman parte de su vida cotidiana. Por ello, en este trabajo se propone la incorporación de las Tecnologías de la Información y la Comunicación (TIC), junto con otros materiales de calidad obtenidos en la web, como soporte de información de los contenidos docentes. El objetivo principal es que las herramientas propuestas permitan profundizar en la materia de forma más atractiva y amena, así como fomentar la
} 
comunicación entre alumnos y profesores. Este modelo se ha implementado dentro de las clases de Matemáticas I del Grado en Ingeniería Electrónica Industrial y Automática, tanto en la teoría como en las prácticas de aula e informáticas.

Palabras clave: TIC, Recursos tecnológicos, Aprendizaje de matemáticas, Materiales docentes dinámicos

\section{Introducción}

La enseñanza universitaria tiene la doble finalidad de preparar profesionales cualificados y completar su formación integral. Esta formación se materializa en la adquisición de un conjunto de conocimientos, habilidades y estrategias adecuados a cada perfil, donde el binomio enseñanza-aprendizaje tiene un papel protagonista.

En un contexto donde nuestro objetivo es facilitar el aprendizaje significativo del alumnado, no basta ser un experto en los contenidos que se imparten, ya que la forma de exponerlos y de trabajar con ellos también es muy importante. $\mathrm{Y}$ es, en este punto, donde una adecuada elección de la metodología docente nos facilitará la tarea.

Para ello, hemos de ser conscientes que en el aula conviven una gran variedad de estilos de aprendizaje (Lago et al. 2008), conocimientos previos y motivaciones diferentes, por lo que con el objetivo de respetar al máximo esta diversidad, nuestra propuesta consiste en utilizar una variedad razonable de actividades y materiales, que nos permita adecuar nuestra docencia al mayor número de alumnos posible. Centrando nuestra atención en recursos habituales para nuestros alumnos, como son el uso de las Tecnologías de la Información y la Comunicación (TIC) y los materiales disponibles en las redes sociales. Ya que la utilización de este tipo de recursos genera cierta inseguridad a la hora de incorporarla en nuestras aulas, tanto por su rápida evolución, como por su cuestionable fiabilidad. Sin embargo, consideramos que esto no debe impedir que nuestros alumnos aprovechen sus ventajas, entre otras, una mayor libertad de elección, en base a sus necesidades y gustos, una mejor integración de los conocimientos, gracias a la visualización de sus aplicaciones, y la aportación de competencias fundamentales, en la realidad globalizada en la que vivimos, como la innovación o la creatividad.

Por otro lado, como nativos digitales, nuestros alumnos encuentran en el manejo de las TIC y las redes sociales su forma natural de comunicarse. Esto hace que estén acostumbrados a obtener la información que necesitan en varios formatos diferentes, en general interactivos, 
y de forma inmediata y bastante dispersa. De forma que habitualmente les cuesta focalizar su atención, especialmente en los formatos más tradicionales utilizados en clase, y esto produce una desmotivación que se traduce, en general, en una pérdida de interés y de atención en las asignaturas.

Por tanto, para paliar en lo posible estos inconvenientes, pretendemos dinamizar nuestra docencia introduciendo en nuestra aula este tipo de materiales (Fernández et al. 2006).

Puesto que en algunas ocasiones no disponemos del tiempo, ni los conocimientos para generar un material suficientemente atractivo, nuestra propuesta es apoyarnos en materiales ya existentes, disponibles en la web, como canales de Youtube de otros profesores y/o especialistas en la materia, o bien en repositorios cuyos materiales hayan pasado una revisión y tengan una calidad reconocida, como por ejemplo RiuNet en la UPV (entre otros muchos), ya que cada vez más alumnos utilizan contenidos, principalmente audiovisuales, presentes en las redes sociales, para prepararse las asignaturas. Incluir este tipo de materiales, bajo nuestra supervisión, no solo garantiza la calidad de los mismos, sino que les da las claves sobre cómo utilizarlos de manera eficaz, a través de la visualización en el momento más adecuado.

El artículo está estructurado de la siguiente forma, en la primera sección presentamos los objetivos del trabajo, en la segunda el desarrollo de la innovación, con subsecciones dedicadas a la descripción de nuestra propuesta, en los distintos entornos de la asignatura: la teoría de aula, las prácticas de aula y las prácticas informáticas. Seguidamente, en la tercera sección 3, comentamos los resultados de la encuesta que hemos pasado a los alumnos para conocer su opinión al respecto y finalmente, en la cuarta y última sección, presentamos las conclusiones del trabajo.

\section{Objetivos}

Con esta propuesta, pretendemos mejorar la integración de los recursos audiovisuales obtenidos en la web con actividades en las que tanto el profesor como los alumnos se sientan cómodos y disfruten de los beneficios que las TIC ofrecen, complementando de esta manera un enfoque más tradicional de la docencia y reforzando el proceso de aprendizaje de los alumnos.

Así, los objetivos que pretendemos alcanzar son los siguientes:

- Poner a disposición de los alumnos materiales de calidad relacionadas con la materia, lo más variados e interactivos posible como, por ejemplo, vídeos, "screencast", “applets", laboratorios virtuales, etc., bien creándolos nosotros mismos, o bien apoyarnos en los ya existentes en las redes sociales o repositorios, 
para dinamizar el aprendizaje de los alumnos y desarrollar su espíritu crítico a la hora de consultar nuevas fuentes, ya que lo harán, en la mayoría de los casos, lo consideremos adecuado o no.

- Plantear actividades con ellos que nos permitan presentar la información en un formato más atractivo que los tradicionales textos y documentos, acercándonos a la realidad de nuestros alumnos, y añadiendo así un factor motivador que hace las clases más amenas.

- Trabajar fuera del aula aspectos mecánicos que pueden resultar tediosos o repetitivos dentro de ella, y así atender mejor a la diversidad del alumnado, fomentando un aprendizaje más adaptado en nuestra aula.

\section{Desarrollo de la innovación}

La propuesta de este trabajo se ha desarrollado en la asignatura Matemáticas I del Grado en Electrónica Industrial y Automática, impartido en la Escuela Técnica Superior de Ingeniería del Diseño (ETSID) de la Universitat Politècnica de València (UPV).

Matemáticas I es una asignatura obligatoria de 9 créditos ECTS, repartido en 4.5 de teoría, 2 de prácticas de aula y 2.5 de prácticas informáticas. Los contenidos que se trabajan en ella son los habituales en una asignatura básica de matemáticas, con contenidos de cálculo y álgebra lineal, que detallamos a continuación:

Unidad 1. Cálculo diferencial de funciones de una y varias variables

Tema 1. Números complejos

Tema 2. Cálculo diferencial de funciones de una variable. Funciones hiperbólicas

Tema 3. Funciones de varias variables

3.1. Dominio y curvas de nivel

3.2. Límites y continuidad

3.3. Diferenciabilidad

Tema 4. Derivación de funciones compuestas e implícitas

Tema 5. Extremos de funciones de varias variables

Prácticas de laboratorio asociadas a la Unidad 1:

Práctica 1: Introducción al Mathematica. Operaciones y matrices

Práctica 2: Números complejos. Resolución de ecuaciones y sistemas

Práctica 3: Funciones de una variable: gráficas, límites y derivadas

Práctica 4: Funciones de varias variables: gráficas, límites y derivadas

Práctica 5: Extremos de funciones de varias variables (libres y condicionados)

Unidad 2. Cálculo integral de funciones de una y varias variables 
Tema 1. Cálculo integral de funciones de una variable

1.1. Primitivas. Métodos elementales de integración

1.2. Integral definida y aplicaciones

Tema 2. Cálculo integral de funciones de varias variables

2.1. Integrales dobles

2.2. Integrales triples

2.3. Aplicaciones: cálculo de volúmenes, masas

Tema 3. Integral curvilínea de campos escalares y de campos vectoriales

3.1. Aplicaciones: longitudes de curvas, masas, trabajo

Prácticas de laboratorio asociadas a la Unidad 2:

Práctica 6: Primitiva e integrales definidas

Práctica 7: Aplicaciones de la integral definida

Práctica 8: Integrales múltiples y aplicaciones

Práctica 9: Integración curvilínea y aplicaciones

Unidad 3. Álgebra

Tema 1. Sistemas de ecuaciones lineales. Polinomios interpoladores

Tema 2. Matrices y determinantes. Mínimos cuadrados

Tema 3. Espacios vectoriales

Tema 4. Diagonalización de matrices

Prácticas de laboratorio asociadas a la Unidad 3:

Práctica 10: Sistemas de ecuaciones lineales. Interpolación

Práctica 11: Matrices y determinantes. Mínimos cuadrados

Práctica 12: Espacios vectoriales y diagonalización de matrices

En los siguientes apartados, detallamos como hemos implementado los materiales obtenidos en la web, tanto en la teoría de aula como en las prácticas de aula y las prácticas informáticas.

\subsection{Teoría de aula}

Aunque la clase magistral participativa es nuestra propuesta para exponer en el aula los contenidos teóricos, donde para cada tema elaboramos una presentación con diapositivas, que nos sirve de apoyo, donde detallamos los objetivos, contenidos del tema y su distribución. En esta presentación, enlazamos con páginas web, recursos multimedia y representaciones gráficas, que ejemplifican y dan contexto a los nuevos conceptos, con el objetivo de atender tanto a los distintos niveles de conocimientos previos de nuestros alumnos, como a la variedad de estilos de aprendizaje y motivaciones que tienen. 
Esto nos conduce a introducir cada tema relacionando sus contenidos con otros que les resulten familiares, unificando nomenclaturas y notaciones, para construir el aprendizaje en la materia sobre unas bases sólidas.

Por otro lado, proporcionamos además de la clásica bibliografía sobre los temas tratados, vídeos adecuados para perfeccionar y ampliar el conocimiento en la materia, tanto de canales de YouTube como del repositorio RiuNet, entre otros.

Como ejemplo, podemos citar el vídeo sobre la superficie de revolución La Trompeta de Gabriel, que el reconocido divulgador y profesor de la Universidad de la Rioja E. Sáenz de Cabezón tiene en su canal de YouTube Derivando, patrocinado por la UPV: https://www.youtube.com/watch?v=Q4RLtRlz-4A.

O interesantes vídeos sobre la interpretación geométrica de las integrales dobles y el cambio a polares, que el profesor de física E. Khutoryansky tiene en su canal de YouTube Physic Videos: https://www.youtube.com/embed/GHBMiscPE-g.

Además, de los polimedias relacionados con nuestro temario, elaborado por diversos profesores del Departamento de Matemática Aplicada (DMA) y presentes en el repositorio RiuNet de la UPV, como este sobre cambio de variable en integrales múltiples de M. Trujillo: https://media.upv.es/player/?id=b29107b5-c6c2-8244-bd29-11ba034fccbb.

\subsection{Prácticas de aula}

Consideramos que la resolución de ejercicios y problemas es una buena metodología para afianzar, contextualizar y aplicar los contenidos teóricos de la clase magistral en las prácticas de aula, donde siempre es interesante que los ejercicios estén lo más relacionados posible con los intereses del alumnado. En cada unidad preparamos un listado de problemas, que los alumnos deben resolver de forma autónoma, además de los que realizan en el aula.

En este caso, fomentamos que los alumnos resuelvan los ejercicios y los compartan con sus compañeros, a través de la plataforma docente PoliformaT, previa revisión por parte del profesor. De esta manera, pueden desarrollar mejor su criterio a la hora de buscar otros problemas resueltos, ya que la consulta de ejercicios y explicaciones en la web, a través de canales de YouTube o páginas web orientadas a estos temas, es una práctica muy habitual entre los alumnos. Canales de YouTube muy populares en este sentido son Unicoos (https://www.youtube.com/user/davidcpv) o www.julioprofe.net, entre otros, además de varias páginas web donde se ofrecen clases particulares o se comparten cursos como SharingAcademy (https://sharingacademy.com) o Tutellus (https://www.tutellus.com). 
Estas herramientas favorecen, principalmente, a alumnos que presentan alguna carencia en su base matemática, bien porque hace tiempo que no trabajan la materia, bien porque proceden de estudios que no requieren este tipo de conocimientos previos.

En algunos casos, el material elaborado por los alumnos para completar los materiales del profesor es de una gran calidad y puede resultar muy útil al resto de sus compañeros. Además, actualmente, las redes sociales ofrecen herramientas de todo tipo que permiten compartir apuntes y compendios de explicaciones con ejemplos y ejercicios resueltos desde el punto de vista del alumno, como por ejemplo la plataforma online Woulah (https://www.wuolah.com), enlaces de descarga compartidos desde grupos formados en nubes informáticas como Google Drive, o Dropbox, entre otros tantos medios donde se comercializa con este tipo de contenido que, en ocasiones con gran rigor, ayudan al alumnado a alcanzar, en mayor o menor medida, los objetivos de conocimiento previstos.

En este sentido, como consecuencia de la participación del alumnado en el desarrollo de su propia formación a este nivel, podremos encontrar en un futuro próximo una aplicación para el móvil y un libro de apoyo, ambos desarrollado desde el punto de vista de los estudiantes del Grado en Ingeniería Electrónica Industrial y Automática de la ETSID, con el respaldo y supervisión del Departamento de Matemática Aplicada. Este es un ejemplo de colaboración entre alumnado y profesorado, que pretende arrojar luz y servir de apoyo al aprendizaje, puesto que en ocasiones el docente, dados sus conocimientos y posición académica muy lejana a la del alumno, pasa por alto conceptos puntuales porque los considera ya adquiridos, cuando en muchas ocasiones no es así, y que son clave para entender los contenidos del temario.

Por otro lado, la diversidad de procedencias y niveles académicos dispares en los alumnos hace que los materiales de este tipo faciliten, que todos los estudiantes, sean capaces de alcanzar, a su ritmo, los conocimientos requeridos. Por tanto, este tipo de iniciativas, fruto de la colaboración en el aula, constituye un método más, que aporta apoyo a ambas partes.

\subsection{Prácticas informáticas}

El contenido teórico de la asignatura tiene prácticas informáticas asociadas que realizamos con el software Mathematica, programa muy versátil, con sintaxis bastante intuitiva y mucha información en la ayuda, que resulta ser una herramienta de cálculo suficientemente potente para la realización y comprobación de ejercicios y problemas.

En la asignatura de Matemáticas I, utilizamos las prácticas informáticas principalmente para resolver con un software una gran variedad de ejercicios relacionados con teoría de aula, por lo que empezamos cada práctica presentando los comandos del programa que utilizaremos, 
para desarrollar los ejemplos y ejercicios que permitan al alumno tanto afianzar los conocimientos adquiridos en la materia, como mejorar en el manejo del software.

Los alumnos disponen en PoliformaT, antes del inicio de cada tema, de todo el material que utilizamos, ya que en las prácticas de laboratorio utilizamos clase inversa, siguiendo el proyecto Docencia Inversa de la UPV. Esta metodología implica más al alumno en la asignatura y nuestra experiencia al respecto está siendo satisfactoria, como muestran los resultados recogidos en (Coll et al., 2016, Coll et al. 2017).

Así, cada práctica consta de tres fases:

- Primera fase o preparación inicial por parte del alumno, donde debe trabajar el contenido de la práctica de forma autónoma, antes de asistir al laboratorio. Para facilitarle esta labor, utilizamos vídeos tipo "screencast", es decir, capturas en vídeo de la pantalla del ordenador, donde se presentan los comandos que vamos a utilizar, así como la forma de aplicarlos en varios ejemplos, además de algunos ejercicios propuestos que el alumno debe resolver para autoevaluarse. En este caso, los vídeos utilizados son del Curso Mathematica del canal de YouTube del profesor de Matemáticas J. L. Tabara Carbajo: https://www.youtube.com/playlist?list=PLilnwC7Bci64kNIvqPaFE4KmjDtkEpnSj

- Segunda fase, correspondiente a una primera sesión presencial en el aula informática, donde hacemos un breve repaso de los contenidos, para ayudar a superar las dificultades que los alumnos hayan encontrado al trabajar la práctica de forma autónoma. Una vez resueltas las dudas, planteamos ejercicios para resolver en grupo, de manera que los alumnos puedan debatir y consensuar la mejor solución con la supervisión del profesor, como vemos en la Figura 1.

- Una tercera fase o segunda sesión presencial en el aula, donde planteamos ejercicios que los alumnos tienen que resolver de forma autónoma mediante la herramienta Exámenes de PoliformaT.

Cada una de estas fases queda reflejada en la plataforma PoliformaT, a través del apartado de exámenes o de tareas, según el caso, donde los alumnos realizan las pruebas y envían el archivo final con el trabajo realizado.

De esta manera, el uso de las TIC facilita la autoevaluación, así como la evaluación compartida (o coevaluación) fel alumnado. Dos herramientas que resultan muy útiles a la hora de dotar a la evaluación de un aspecto formativo, más allá de la mera calificación de la materia, ya que aportan información sobre los progresos y deficiencias observados en el aprendizaje, dando la oportunidad de mejorar. 


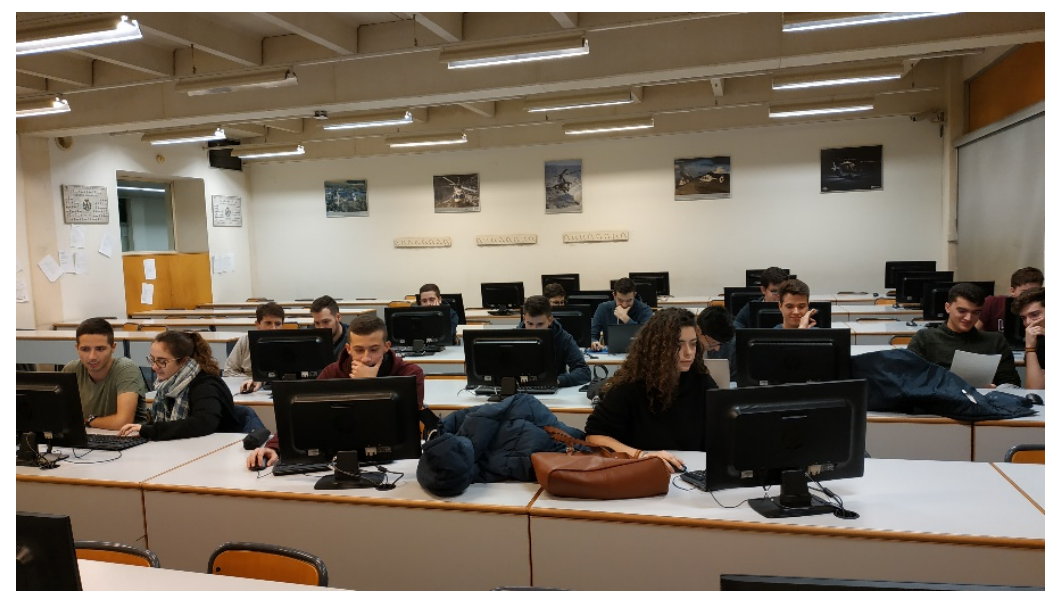

Fig. 1 Alumnos trabajando en el aula de prácticas

\section{Resultados}

Para analizar la necesidad de facilitar al alumno estos materiales docentes, así como su introducción progresiva en la metodología de la asignatura, hemos realizado una breve encuesta tomando como muestra tres grupos de prácticas, correspondientes a un grupo de teoría y práctica de aula de Matemáticas I del Grado en Ingeniería Electrónica Industrial.

En particular la encuesta ha sido rellenada por 34 alumnos de los 64 alumnos matriculados en el grupo.

La primera parte de la encuesta está destinada a analizar qué tipo de materiales, presentes en el apartado de Recursos del PoliformaT de la asignatura, les resultan más útiles a la hora de preparar la asignatura. Para ello, les hemos pedido que indiquen el porcentaje de utilidad que obtienen de cada tipo de material, a la hora de estudiar nuestra asignatura, siendo los recursos ofertados: Apuntes teóricos, Transparencias, Ejercicios propuestos, Ejercicios resueltos y Vídeos y recursos interactivos.

Las medias de los porcentajes obtenidos en cada uno de los tipos de recursos se presentan con un diagrama de sectores en la Figura 2, donde apreciamos que no hay ningún tipo de recurso que los alumnos vean de mayor utilidad que los demás, sino que más bien muestra que todos, con algunas pequeñas diferencias, les resultan útiles en cierta medida con la excepción de los vídeos y recursos interactivos. Esto probablemente se debe a que muchos alumnos no utilizan este tipo de material para la preparación de la teoría y la práctica de aula. También se puede observar, que lo que más interés tiene para ellos son los ejercicios resueltos, ya que les sirve como práctica y entrenamiento para los exámenes. Y que los ejercicios propuestos son los que resultan de menor utilidad, puesto que no conocen la 
solución y no se sienten demasiado motivados a intentar resolverlos. Entre los recursos teóricos (Apuntes y Transparencias) apenas existen diferencias y ambos resultan una parte importante como soporte docente.
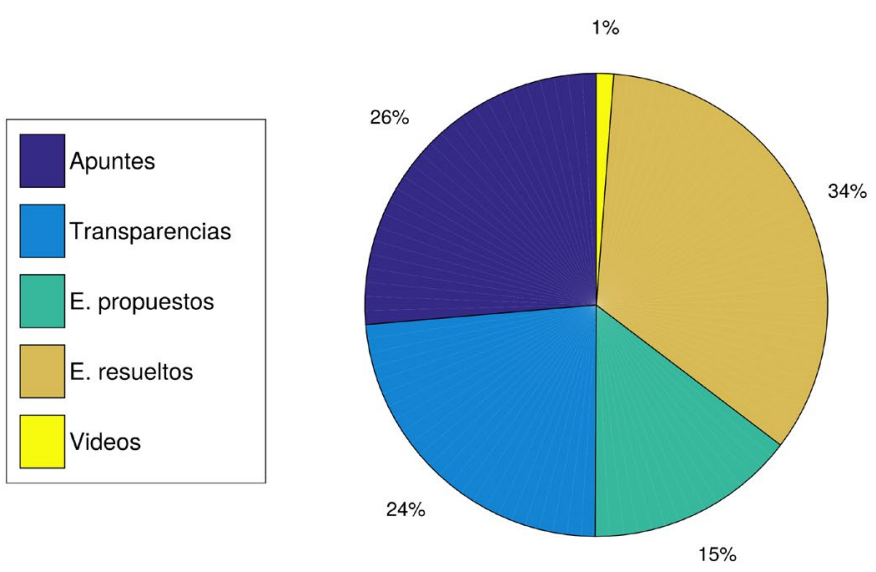

Fig. 2 Porcentajes de utilidad de los recursos que se les ofrecen a los alumnos

La segunda parte de la encuesta está centrada en estudiar la utilidad de los videos propuestos en la parte de prácticas informáticas tal y como se describe en la sección 2.3.

El estudio se ha realizado sobre un grupo de teoría donde se encuentran alumnos que no han utilizado los videos para preparar las prácticas y otros que sí. En concreto, 17 de los alumnos encuestados no han tenido disponible dicho material y el resto, otros 17 , lo han tenido accesible.

Por ello, hemos formulado la pregunta relativa a este apartado dependiendo del grupo de prácticas al que hayan asistido. A los encuestados que disponían del recurso tecnológico, les hemos consultado sobre su utilidad para la preparación de las prácticas, mientras que en los que pertenecían a los grupos de prácticas que no ofrecían este contenido, la pregunta estaba orientada a saber si les hubiese gustado tener disponible también este tipo de material.

Los resultados de estos dos grupos están representados en la Figura 3 y, a la vista de ambos diagramas, es razonable pensar que a los alumnos les resultan útiles los videos en la preparación de las prácticas, ya que los que no utilizaron este tipo de material, hubieran estado bastante interesados en tenerlos disponibles. 


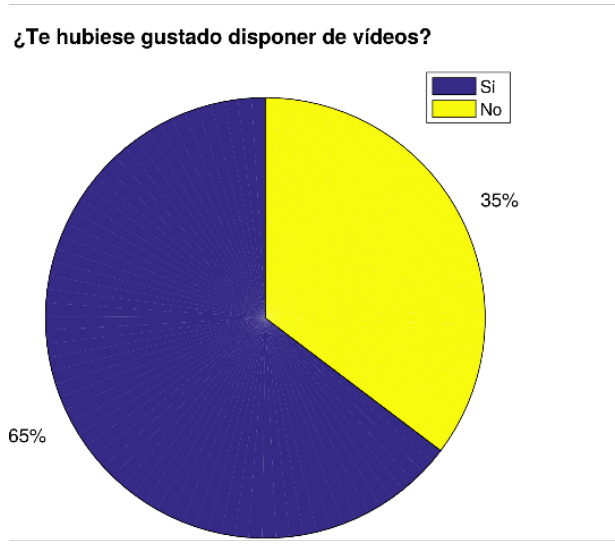

Fig. 3 Resultados sobre la utilidad de los vídeos en las prácticas informáticas

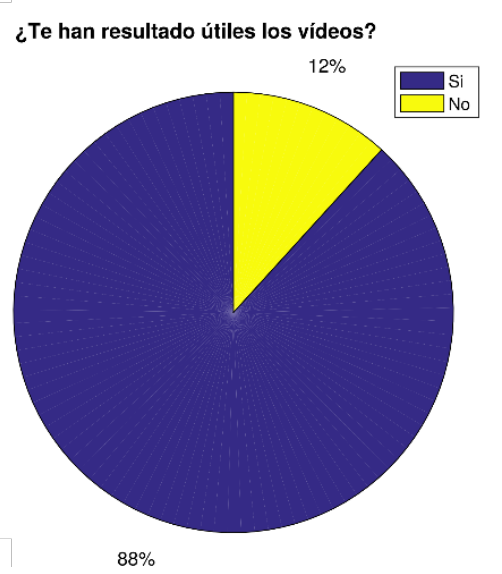

Por último, hemos preguntado a los alumnos, en modo de respuesta abierta, el tipo de materiales que les gusta consultar por su cuenta a la hora de estudiar la asignatura, además de los facilitados por el profesor. Las respuestas mayoritarias, además de los ya comentados ejercicios resueltos, transparencias y documentos presentes en PoliformaT, son exámenes de años anteriores y apuntes tomados por ellos mismos en clase.

Cabe destacar también, que muchos de ellos hacen uso de la página web Unicoos (https://www.unicoos.com), Vitutor (https://www.vitutor.com), así como de canales temáticos de YouTube. Es decir, aunque no han valorado demasiado este tipo de materiales en la primera pregunta, queda claro aquí que los alumnos buscan los recursos tecnológicos por su cuenta, suponemos que para encontrar los más adaptados a su nivel y gusto personal.

\section{Conclusiones}

Con el fin de adecuarnos a la realidad actual, atender de forma más personalizada las necesidades de nuestros alumnos y dinamizar así nuestra asignatura, incluimos en nuestra metodología actividades utilizando materiales de calidad, disponibles en la web, con el objetivo de aprovechar los beneficios, tanto didácticos como motivadores, que las TIC y estos novedosos formatos nos ofrecen.

De esta forma, adaptamos nuestra docencia a un lenguaje y formato más acorde con la realidad de nuestros alumnos, como parece indicarnos la buena acogida que han tenido los vídeos de YouTube utilizados en las clases prácticas. En posteriores análisis, una vez tengamos las notas finales, esperamos que nuestra propuesta refleje también una influencia positiva en el aprendizaje de los alumnos, no solo en la parte de prácticas informáticas, sino también en la teoría y las prácticas de aula. 


\section{Referencias}

COLL, C., GINESTAR, D. SANABRIA, E., SANCHEZ, E. (2016). "Introducir Flip Education en nuestras aulas. Una experiencia en las prácticas de Matemáticas" en XIV Jornadas de Redes de Investigación en Docencia Universitaria. ICE. Universidad de Alicante.

COLL, C., GINESTAR, D. SANABRIA, E., SANCHEZ, E. (2017). "La metodología de clase inversa en la UPV: Una experiencia en el laboratorio de matemáticas" en In-Red 2017 III Congreso Nacional de innovación educativa y de docencia en red. Universitat Politècnica de València.

FERNÁNDEZ, B., SUÁREZ, L., ALVÁREZ, E. (2006). "El camino hacia el Espacio Europeo de Educación Superior: deficiencias metodológicas y propuestas de mejora desde la perspectiva del alumno." en Aula Abierta. 88, p. 85-105.

KHUTORYANSKY, E. (2016) "Double integrals and Polar integrals: Explained with 3D

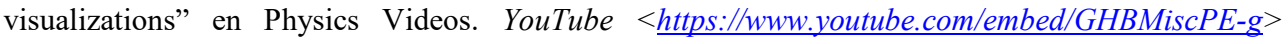
[Consulta: 25 de marzo de 2019]

LAGO, B., COLVIN, L., CACHEIRO, M. (2008). "Estilos de Aprendizaje y Actividades Polifásicas : Modelo EAAP.', en Revista Estilos de Aprendizaje, 2, vol. 2.

SAENZ DE CABEZON, E. (2018) “¡El apocalipsis matemático! La trompeta de Gabriel” en Derivando. YouTube <https://www.youtube.com/watch? $v=Q 4 R L t R l z-4 A>$ [Consulta: 25 de marzo de 2019]

TABARA CARBAJO, J. L. (2014) “Curso Mathematica”. YouTube

$<$ https://www.youtube.com/watch? $v=O 2 j h v b q V u G k \& t=231 s>$ [Consulta: 25 de marzo de 2019]

TRUJILLO GUILLEN, M. (2011) “Cambio de variable en integrales múltiples. Coordenadas polares” en RiuNet Repositorio UPV.

Disponible en <https://polimedia.upv.es/visor/?id=b29107b5-c6c2-8244-bd29-11ba034fccbb>

[Consulta: 25 de marzo de 2019]

WOLFRAM, S. Mathematica de wolfram research. $<$ https://www.wolfram.com/mathematica $>$ 